\title{
Biodiversity and Distribution of Gastropods at Seagrass Meadow of Balangdatu Waters Tanakeke Island South Sulawesi Indonesia
}

\author{
Magdalena Litaay, Marwa Deviana and Dody Priosambodo
}

Department of Biology, Faculty of Mathematics and Natural Sciences, Hasanuddin University, Makassar, Indonesia

\section{Abstract}

The research about the biodiversity of gastropod has been conducted in seagrass meadow of Balangdatu waters, Tanakeke Island, South Sulawesi. The research aims to assess the diversity of gastropod species in Balangdatu waters. Sampling was conducted using quadrate transect method systematically. Three replicates of transect were applied for each station. The result indicates there were 34 species of gastropods from 14 genera and 14 families were found. Diversity index from every station varies from 1,661 to 2,899 . These values range from low to moderate. The diversity, Evenness, and dominance indices showed that Balangdatu waters still in good condition and sustain habitat for gastropod.

\section{Article History}

Received 12 October 2017

Accepted 29 December 2017

Keyword

Biodiversity

Marine mollusca

Seagrass meadow

Spermonde

\section{Introduction}

Gastropods are known as snails that has more diverse members in the phylum of Mollusks. Main characteristics of this group are single shell, well-developed head and its body is completed by tentacle, eyes and radula. Some gastropods are economics important due a valuable shell for ornament materials and souvenirs or meat as protein sources for human. Therefore, gastropods are recognized as potency group that can contribute to coastal community commodity welfare and fishery productivity at coastal areas. Amongst mollusks group, Gastropods is a successful group in term of widely distributed. They can be found in almost all habitats including the terrestrial, freshwater, and deep sea. Gastropods are also found living in different coastal ecosystem including mangrove, seagrass meadows and coral reefs. (Tomascik et al., 1997).

Coastal communities utilized seagrass meadows for different purposes such as fishing ground, collecting snail as seaweeds aquaculture. On coastal aquaculture practice, this area is often cleaned from unwanted organisms including parasite of seaweed. This action will contribute to damage of seagrass meadows. Hence, seagrass ecosystems are a threat worldwide. Many studies have been conducted on Mollusca around Indonesia, but still less studied on seagrass meadows of South Sulawesi (Litaay 2006, Kusnadi 2008, Litaay et al., 
2010, Arbi 2011, Metungun et al., 2011). On of coastal area in South Sulawesi province that has seagrass meadows that used for seaweed cultivation is Tanakeke Island of Takalar Regency. High activity of human along Tanakeke coastal area to some extent will contribute to a change in water quality that will disturb seagrass meadow. Information on associate biota to seagrass meadows from this area is limited. Information on biodiversity of associate biotas to different ecosystems is needed to support sustainable used of coastal and marine resources. The aim of the present study on gastropods diversity that associates with seagrass meadows of Balangdatu water of Tanakeke Island South Sulawesi is needed to support sustainable use these resources.

\section{Materials and Methods}

\section{Sampling Site}

Sampling site was determined based on water condition, topography, habitat type, distribution of seagrass meadows and community activity along the coast of Balangdatu. We divided sampling sites into three areas as follows:

Site 1 : close to lagoon, substrate rubble, seaweed cultivation area, Site II : seaweed cultivation areas, substrate sand,

Site III : close to human settlement, substrate muddy sand, many seaweeds cultivation

\section{Sampling Methods}

Sampling method of transect combination with the plot was used systematically during gastropods collection (Modified English et al., 1997, Hemminga and Duerte 2000, Priosambodo 2014). Three transects and two replicates were applied at each sampling site. Each transect was divided into 5 plot. A transect was placed at seagrass offshore until reach top end that seagrass was found. Balangdatu has $4,800 \mathrm{~m}$ coastal line in which seagrass meadows areas, divided into three sites: Sta. I: $150 \mathrm{~m}^{2}$, Sta. 2: $300 \mathrm{~m}^{2}$ and Sta. 3: $600 \mathrm{~m}^{2}$. Gastropods in all transect were collected, cleaned and preserved into $70 \%$ alcohol, brought to the laboratory for further identification. Samples were photographed in situ before being preserved. Identification was done based on main characters (Dharma, 2005).

\section{Environment Parameters}

Environment parameters including salinity, temperature, $\mathrm{pH}$, dissolved oxygen and sediment type were measured in situ, respectively.

\section{Data Analysis}

Ecology indices included absolute density, species diversity, evenness, dominance index, and dispersal pattern was applied in this study. Data was analysed according to formulas as follows (Odum 1993):

a. Density $(\mathrm{Di})=\mathrm{ni} / \mathrm{A}$, where:
$A \quad=$ Density species i;
$\mathrm{ni}=$ Total No of ind species $\mathrm{i}$;
$\mathrm{L} \quad=$ Total area of plot

b. Simpson Dominance Index $\mathrm{C}=\Sigma(\mathrm{ni} / \mathrm{N}) 2$, where :
$\mathrm{ni}=$ No of ind species $-\mathrm{i}$
$\mathrm{N}=$ Total no of ind all species 


\section{Criteria :}

$$
\begin{array}{ll}
0<C \leq 0,5 & =\text { Low } \\
0,5<C \leq 0,75 & =\text { Moderate } \\
0,75<C \leq 1,00 & =\text { High }
\end{array}
$$

c. Shanon-Wiener Diversity Index $\mathrm{H}=-\sum(\mathrm{ni} / \mathrm{N}) \ln (\mathrm{ni} / \mathrm{N})$, where :

$$
\begin{aligned}
& \text { ni }=\text { Number of spesies }-\mathrm{i} \\
& \mathrm{N}=\text { Total number of species } \\
& \mathrm{H}^{\prime}=\text { Diversity Index }
\end{aligned}
$$

\section{Criteria :}

$$
\begin{aligned}
& 0<H^{\prime} \leq 2,0=\text { Low } \\
& 2<H^{\prime} \leq 3,0=\text { Moderate } \\
& 3,0<H^{\prime} \leq 4,0=\text { High }
\end{aligned}
$$

d. Morisita Index Id $={ }_{n} \frac{\sum X^{2}-N}{N(N-1)}$, where :

$$
\begin{array}{ll}
\mathrm{n}=\text { No of plot/transect } & =\sum \mathrm{F}(\mathrm{X}) \\
\mathrm{N}=\text { Total no of indiv inside plot/transect } & =\sum[\mathrm{F}(\mathrm{X})](\mathrm{X}) \\
\sum \mathrm{X}^{2}=\text { quadrate no of indiv inside plot } & =\sum[\mathrm{F}(\mathrm{X})]\left(\mathrm{X}^{2}\right)
\end{array}
$$

\section{Criteria :}

Id $<1,0=$ Randomly distributed

Id $=1,0=$ Uniformly distributed

Id $>1,0=$ Clumped distributed

\section{Results and discussion}

\section{Species Composition}

We found 14 families and 14 genera of Gastropods that consists of 34 species. These species are Aluco aluco, Chicoreus capucinus, Conus eximus, Conus ferrugineus, Conus magus, Conus varius, Cymbiola vesvertillo, Cypraea annulus, Cypraea talpa, Cypraea tigris, Cypraea vitellus, Engina alveolata, Engina armilata, Engina concinna, Lambis lambis, Lambis truncata, Littoraria scabra, Nassarius arcularius, Nassarius jacksonianus, Nassarius olivaceus, Nassarius reeveanus, Nassarius stolatus, Nassarius venustus, Nerita squamulata, Oliva tigridella, Oliva taeniata, Polinices mammilla, Polinices melanostomus, Pyrene decussata, Rhinoclavis vertagus, Strombus labiatus, Strombus urceus, Thais tuberosa, Trochus californicus.

Amongst 34 species of gastropods, 7 species was found at St. I, 24 species was at Sta. II, and 13 species at Sta. III. In terms of species composition, Gastropoda from family Nassaridae genus Nassarium are more abundance with 6 species. On the other hand, family Ciiridae, Columbellidae, Littorinidae, Neritopsidae, and Volutidae only consist of 1 species. This study found Strombus urceus occurred frequently, counted for $41.379 \%$ from total occurrence. 


\section{Density}

The result shows that absolute density of gastropods at each station are follows: Sta. I 0.06-0.8 ind $/ \mathrm{m}^{2}$, Sta. II 0.06-0.5 ind $/ \mathrm{m}^{2}$, and Sta. III 0.06-2.22 ind $/ \mathrm{m}^{2}$ (Table 1). Strombus urceus shows highest occurrence at Sta. I (41.379\%) (Table 2). This in accordance with study done by Arbi (2001). He found that Strombus urceus was one of gastropods that distributed widely at Talise waters of North Sulawesi. The lowest density was shown by Chicoreus capucinus and Thais tuberosa. Seagrass medows of Balangdatu waters were situated nearby mangrove and coral reefs may allow some gastropods that origin from coral reef areas as hard substrate were found at seagrass areas. The last two species Chicoreus capucinus and Thais tuberosa that present at seagrass meadows may due to tidal at that location. Supriharyono (2000) argued that current have contributed significantly to a dispersal or movement from benthic fauna from one ecosystem to others.

Table 1. Occurrences of gastropods at three sampling sites

\begin{tabular}{|c|c|c|c|c|}
\hline No & Species & Sta. 1 & Sta. 2 & Sta. 3 \\
\hline 1 & Aluco aluco & - & + & + \\
\hline 2 & Chicoreus capucinus & - & + & - \\
\hline 3 & Conus eximus & - & + & - \\
\hline 4 & Conus ferrugineus & + & + & + \\
\hline 5 & Conus magus & - & + & - \\
\hline 6 & Conus varius & - & + & - \\
\hline 7 & Cymbiola vesvertillo & + & - & + \\
\hline 8 & Cypreae annulus & - & + & - \\
\hline 9 & Cypreae talpa & - & + & - \\
\hline 10 & Cypreae tigris & - & - & + \\
\hline 11 & Cypreae vitellus & + & + & + \\
\hline 12 & Engina alveolata & - & + & - \\
\hline 13 & Engina armilata & - & - & + \\
\hline 14 & Engina concinna & - & + & - \\
\hline 15 & Lambis lambis & + & - & + \\
\hline 16 & Lambis struncata & - & + & - \\
\hline 17 & Littoraria scabra & - & + & - \\
\hline 18 & Nassarius arcularius & - & + & - \\
\hline 19 & Nassarius jacksonianus & - & + & - \\
\hline 20 & Nassarius olivaceus & - & + & - \\
\hline 21 & Nassarius reeveanus & - & + & - \\
\hline 22 & Nassarius stolatus & + & - & - \\
\hline 23 & Nassarius venustus & - & + & - \\
\hline 24 & Nerita squamulata & - & + & - \\
\hline 25 & Oliva tigridella & - & + & + \\
\hline 26 & Olivia taeniata & - & + & - \\
\hline 27 & Polinices mammilla & - & + & - \\
\hline 28 & Polinices melanostomus & - & - & + \\
\hline 29 & Pyrene decussata & - & + & - \\
\hline 30 & Rhinoclavis vertagus & - & - & + \\
\hline 31 & Strombus labiatus & + & - & - \\
\hline 32 & Strombus urceus & + & + & + \\
\hline
\end{tabular}


33 Thais tuberosa - - $\quad-\quad+$

34 Trochus $s p$

$+$

Table 2. Density of Gastropods at seagrass meadows of Balangdatu waters Tanakeke

\begin{tabular}{|c|c|c|c|c|}
\hline \multirow{2}{*}{ No. } & \multirow{2}{*}{ Gastropods } & \multicolumn{3}{|c|}{ Density (ind $/ \mathrm{m}^{2}$ ) } \\
\hline & & Sta. I & Sta. II & Sta. III \\
\hline 1 & Aluco aluco & 0 & 0.200 & 0.533 \\
\hline 2 & Chicoreus capucinus & 0 & 0.066 & 0 \\
\hline 3 & Conus eximus & 0 & 0.266 & 0 \\
\hline 4 & Conus ferrugineus & 0.200 & 0.133 & 0.933 \\
\hline 5 & Conus magus & 0 & 0.200 & 0 \\
\hline 6 & Conus varius & 0 & 0.066 & 0 \\
\hline 7 & Cymbiola vesvertillo & 0.133 & 0 & 0.533 \\
\hline 8 & Cypraea annulus & 0 & 0.666 & 0 \\
\hline 9 & Cypraea talpa & 0 & 0.067 & 0 \\
\hline 10 & Cypraea tigris & 0 & 0 & 0.400 \\
\hline 11 & Cypraea vitellus & 0.333 & 0.133 & 0.133 \\
\hline 12 & Engina alveolata & 0 & 0.466 & 0 \\
\hline 13 & Engina armilata & 0 & 0 & 0.200 \\
\hline 14 & Engina concinna & 0 & 0.333 & 0 \\
\hline 15 & Lambis lambis & 0.133 & 0.000 & 0.066 \\
\hline 16 & Lambis struncata & 0 & 0.066 & 0 \\
\hline 17 & Littoraria scabra & 0 & 0.066 & 0 \\
\hline 18 & Nassarius arcularius & 0 & 0.200 & 0 \\
\hline 19 & Nassarius jacksonianus & 0 & 0.133 & 0 \\
\hline 20 & Nassarius olivaceus & 0 & 0.200 & 0 \\
\hline 21 & Nassarius reeveanus & 0 & 0.066 & 0 \\
\hline 22 & Nassarius stolatus & 0.267 & 0 & 0 \\
\hline 23 & Nassarius venustus & 0 & 0.066 & 0 \\
\hline 24 & Nerita squamulata & 0 & 0.800 & 0 \\
\hline 25 & Oliva tigridella & 0 & 0.533 & 0.133 \\
\hline 26 & Olivia taeniata & 0 & 0.266 & 0 \\
\hline 27 & Polinices mammilla & 0 & 0.400 & 0 \\
\hline 28 & Polinices melanostomus & 0 & 0 & 0.466 \\
\hline 29 & Pyrene decussata & 0 & 0.266 & 0 \\
\hline 30 & Rhinoclavis vertagus & 0 & 0 & 0.133 \\
\hline 31 & Strombus labiatus & 0.067 & 0 & 0 \\
\hline 32 & Strombus urceus & 0.800 & 0.400 & 2.200 \\
\hline 33 & Thais tuberosa & 0 & 0 & 0.066 \\
\hline 34 & Trochus sp & 0 & 0 & 0.066 \\
\hline & Total & 1.933 & 6.058 & 5.862 \\
\hline
\end{tabular}

\section{Species Diversity $\left(\mathbf{H}^{\prime}\right)$}

Diversity index ranged from 1,661 to 2,899 (Table 3). According to Brower et al., (1990) these values are categorized as low to moderate. Low diversity in Sta. I may due to habitat which dominated by rubble. Comparing to substrates at Sta. II and Sta. III, less nutrient available in this Sta. I type of substrate may contribute to species diversity. 
Table 3. Species Diversity Index $\left(H^{\prime}\right)$ and Evenness Index (E)

\begin{tabular}{cccccc}
\hline \multirow{2}{*}{ No. } & \multirow{2}{*}{ Sta. } & \multicolumn{2}{c}{ Species Diversity Index $\left(\mathbf{H}^{\prime}\right)$} & \multicolumn{2}{c}{ Evenness Index (E) } \\
\cline { 3 - 6 } & & Value & Category & Value & Category \\
\hline 1 & I & 1.661 & Low & 0.853 & Stable \\
2 & II & 2.899 & Moderate & 0.912 & Stable \\
3 & III & 2.006 & Moderate & 0.782 & Stable \\
\hline
\end{tabular}

\section{Evenness Index (E)}

This study revealed that sampling site shows stable condition as shown by values of Evenness $0,782-0,912$ (Table 3). Stable community allows species to occupy equally or they show distribution pattern of more or less equal. Environmental condition that relatively same would allow species to widely distributed at Balangdatu waters. Different type of habitats would have influence species distribution in nature. Leaf density of seagrass as habitat is also affected macrozoobenthos including gastropods as reported by Hemminga and Duarte (2000).

\section{Dominance Index (C)}

In the present study, value of dominance Index ranges from 0.0001 to 0.140 (Table 4). This reveals that community in stable condition as dominance index close to zero, as indication that there is no dominant species. Also, competition amongst species may also result in space distribution Odum (1993).

Table 4. Dominance Index (C) and Morisita Index at different sampling sites

\begin{tabular}{clcccccc}
\hline & & \multicolumn{7}{c}{ Indices } \\
\cline { 3 - 8 } No. & & \multicolumn{7}{c}{ Gominance (C) } & \multicolumn{3}{c}{ Morisita (Id) } \\
\cline { 3 - 8 } & & Sta. I & Sta. II & Sta. III & Sta. I & Sta. II & Sta. III \\
\hline 1 & Aluco aluco & 0 & 0.001 & 0.008 & - & 1 & 0.33 \\
2 & Chicoreus capucinus & 0 & 0.0001 & 0 & - & $\sim$ & - \\
3 & Conus eximus & 0 & 0.001 & 0 & - & 1 & - \\
4 & Conus ferrugineus & 0.01 & 0.00048 & 0.025 & 1 & 1 & 0.259 \\
5 & Conus magus & 0 & 0.001 & 0 & - & 1 & - \\
6 & Conus varius & 0 & 0.0001 & 0 & - & $\sim$ & - \\
7 & Cymbiola vesvertillo & 0.004 & 0 & 0.008 & 1 & - & 0.485 \\
8 & Cypaea annulus & 0 & 0.012 & 0 & - & 0.684 & - \\
9 & Cypraea talpa & 0 & 0.0001 & 0 & - & $\sim$ & - \\
10 & Cypraea tigris & 0 & 0 & 0.004 & - & - & 0.413 \\
11 & Cypraea vitellus & 0.029 & 0.0004 & 0.0005 & 0.59 & 1 & -0.06 \\
12 & Ensgina alveolata & 0 & 0.005 & 0 & - & 1 & - \\
13 & Engina armilata & 0 & 0 & 0.001 & - & - & 0.119 \\
14 & Engina concinna & 0 & 0.003 & 0 & - & 0.727 & - \\
15 & Lambis lambis & 0.004 & 0 & 0.0001 & 1 & - & $\sim$ \\
16 & Lambis struncata & 0 & 0.0001 & 0 & - & $\sim$ & - \\
17 & Littoraria scabra & 0 & 0.0001 & 0 & - & $\sim$ & - \\
18 & Nassarius arcularius & 0 & 0.0001 & 0 & - & 1 & - \\
19 & Nassarius jacksonianus & 0 & 0.0004 & 0 & - & 1 & -
\end{tabular}




\begin{tabular}{llcccccc}
20 & Nassarius olivaceus & 0 & 0.001 & 0 & - & 1 & - \\
21 & Nassarius reeveanus & 0 & 0.0001 & 0 & - & $\sim$ & - \\
22 & Nassarius stolatus & 0.019 & 0 & 0 & 1 & - & - \\
23 & Nassarius venustus & 0 & 0.0001 & 0 & - & $\sim$ & - \\
24 & Nerita squamulata & 0 & 0.017 & 0 & - & 0.762 & - \\
25 & Oliva tigridella & 0 & 0.007 & 0.0005 & - & 1 & -0.06 \\
26 & Olivia taeniata & 0 & 0.001 & 0 & - & 0.624 & - \\
27 & Polinices mammilla & 0 & 0.004 & 0.006 & - & 0.611 & - \\
28 & Polinices melanostomus & 0 & 0 & 0 & - & - & 0.523 \\
29 & Pyrene decussata & 0 & 0.001 & 0 & - & 0.624 & - \\
30 & Rhinoclavis vertagus & 0 & 0 & 0.0005 & - & - & 0,060 \\
31 & Strombus labiatus & 0.001 & 0 & 0 & $\sim$ & - & - \\
32 & Strombus urceus & 0.171 & 0.004 & 0.14 & 0.89 & 0.525 & 0.517 \\
33 & Thais tuberosa & 0 & 0 & 0.0001 & - & - & $\sim$ \\
34 & Trochus sp & 0 & 0 & 0.0001 & - & - & $\sim$ \\
\hline
\end{tabular}

\section{Distribution Pattern (Id)}

Generally, gastropods at almost sampling sites shows clumped distribution, although some species were widely distributed. Clumped distribution is shown by value of Morisita Index is close to zero, as described in Table 4. Odum (1993) explained that distribution pattern of species are correlated to feeding habit or feeding mode. Species tends to form a group where more food are available. Beside, external reproduction as characteristics of substrates may also contribute to certain distribution pattern of species such as clumped distribution. Gastropodas such as Cypreae vitellus, Engina armilata, and Olivia tigridella show equal distribution, this pattern indicating that there is competition amongst species, hence space distribution equally.

\section{Environment Parameters}

Environment parameters are shown in Table 5. As seen from Table 5, water temperature ranged from 31.77 to $34.17^{\circ} \mathrm{C}$. These temperatures are in favor to molluscs especially gastropods. Gastropods are having a thick shell, allows this group to survive at quite high temperature. Marine fauna can live normally in water with temperature of $26-32^{\circ} \mathrm{C}$, although several invertebrate fauna can adopt to a high temperature Odum (1993). The present study indicates that salinity of Balangdatu water of about $33.67 \%$ - $34.67 \%$, respectively (Table 5), in which is suitable for macrozoobenthos. Metungun et al., (2011) stated that salinity of $25-40 \%$ is good for marine gastropods. $\mathrm{pH}$ value of sampling site varied from 6.92 to -7.19 (Table 5), these $\mathrm{pH}$ ranged can be tolerated by marine organisms, even though $\mathrm{pH}$ marine water can be varied between 7.5 and 8.4. Gross (1972) in Metungun et al., (2011), most of the aquatic gastropods can live at $\mathrm{pH}$ of 5-9. In terms of dissolved Oxygen (DO), Balangdatu waters contain 4.5 to $5.17 \mathrm{mg} / \mathrm{L}$ (Table 5). Another study at Bone Batang gusung at seagrass meadows showed a similar pattern where DO ranged from 4.6 to $5.5 \mathrm{mg} / \mathrm{L}$. These values support optimal growth for seagrass.

Furthermore, the result of sediment analysis indicates that substrates at sampling sites were categorized as sandy clay (Table 5). Sediment texture differ amongst sampling sites as follows sand $(65.00 \%-67.33 \%)$, dust (11.04-15\%) and clay (4.40-5.30\%). The texture of sediment at seagrass meadows of Balangdatu differs from the common structure at 
Spermonde archipelago that dominated by sand, ruble and gravel (Sawall et al., 2011; 2013), Progeroutz et al., 2012).

Table 5. Environment Parameters at seagrass meadows of Balangdatu waters

\begin{tabular}{lccc}
\hline \multirow{2}{*}{ Parameters } & \multicolumn{3}{c}{ Sampling site } \\
\cline { 2 - 4 } & Sta. I & Sta. II & Sta. III \\
\hline Temperature $\left({ }^{\circ} \mathrm{C}\right)$ & 31.77 & 33.1 & 34.17 \\
pH & 7.19 & 6.92 & 7.19 \\
Salinity (\%o) & 33.67 & 34.67 & 34.67 \\
DO $(\mathrm{mg} / \mathrm{l})$ & 4.5 & 5.13 & 4.97 \\
Substrates & Sandy clay & Sandy clay & Sandy clay \\
\hline
\end{tabular}

\section{Conclusions}

The present study concludes, based on ecology indices, Balangdatu waters of Tanakeke Takalar South Sulawesi is still in good condition and support marine gastropods.

\section{Acknowledgment}

We thank Yusran Nurdin Massa, Regista, Suardi, dan A. Darmawansyah from Yayasan Hutan Biru Indonesia, and local people of Balangdatu Tanakeke for assistance during field work. The Ministry of Education and Higher Degree of Indonesia for providing travel grant for first author to present this work at WCM 2016.

\section{References}

Andy Omar S.B, M. Litaay, N. Anwar. 2006. The Distribution and Abundance of Tropical Abalone (Haliotis spp) at Reef Flat of Bonetambu Island, Makassar. TORANI Spec Publ. 16(2):142-147.

Arbi, U.Y. 2011. Mollusca Community Structure at Seagrass Medow of Talise waters, North Sulawesi. Oseanologi and Limnologi di Indonesia, 37(1):71-89

Brower, J.S, J.H. Zar, N.O. Ende. 1990. Field and Laboratory Methods for General Ecology. Third Edition. Brown.

Cappenberg, A.W. 2014. Megabenthos Community Structure in Pangkajene Waters of Pangkep District South Sulawesi. Zoo Indonesia, 23(2):57-67

Clark, J. 1974. Costal Ecosystem. Ecological Consideration for Management of the Coastal zone. The Conservation Foundation. Washington, DC.

Dharma, B. 1988. Indonesia Shell Part I. Germany.

Dharma, B. 2005. Recent and Fossil. Indonesian Shells. Conch Book. Germany.

English S, C. Wilkinson, V. Baker. 1997. Survey Manual for Tropical Marine Resources.

Australian Institute of Marine Science. Townsville.

Litaay, M. 2006. Macroinvertebrates Associated with Seagrass at Awarange and Labuange Bay South Sulawesi. Torani Spec Publ, 16 (5).

Litaay, M, W. Moka, Ambeng, Fatmanugraha. 2010. Macrozoobenthos as water quality control at Barrang Lompo water. Proceeding of International Conference on Small Island and Coral Reefs (ISSIC)-Sail Banda.

Hemminga, M.A and C.M. Duerte. 2000. Seagrass Ecology. Cambridge University Press. London-United Kingdom. 
Khouw, A.S. 2009. Metode dan Analisa Kuantitatif dalam Bioekologi laut, Pusat Pembelajaran dan Pengembangan Pesisir dan Laut (P4L). Bogor.

Kusnadi, K. 2008. Inventrisasi Jenis dan Potensi Moluska Padang Lamun di Kepulauan Kei Kecil Maluku Tenggara. Biodiversitas, 9 (1):30-34,

Metungun J, Juliana, Y. Mariana. 2011. Gastropods density at seagrass meadows at waters of UN strait at South East Moluccas. Oseanologi and Limnologi In Indonesia, ISBN: 978-602$98439: 225-231$

Odum, E.P. 1993. Ecology. Gadjah Mada University Press. Yogyakarta

Priosambodo, D. 2014. Baseline Survey Manual and Data analysis for Seagrass. Yayasan Hutan Biru Indonesia. Makassar.

Progeroutz C, Asmus H, Ahnelt H, Kneer D, Litaay M. 2012. The influence of Canopy Structure and Tidal Level on Fish Assemblages in Tropical Southeast Asian Seagrass Meadows. Eustarine Coastal and Shelf Science, 107(2):58-68.

Sawall Y, Teichberg M, Seemann J, Litaay M, Jompa J, Richter C. 2011. Nutritional Status and Metabolism of the Coral Stylophora ubseriata along a Eutrophication Gradient in Spermonde Archipelago (Indonesia). Coral Reefs, 30: 841-853.

Sawall Y, C. Richter, J. Jompa, M. Litaay. 2013. Coral Recruitment and Potential Recovery of Eutrophied and Blast Fishing Impacted Reefs in Spermonde Archipelago, Indonesia. Marine Pollution Bulletin, 74(1):374-382.

Supriharyono. 2000. Conservation and Management of Natural Resources in Tropical Coastal Areas. PT. Gramedia Pustaka Utama, Jakarta [In Indonesia]

Tomascik T, A.J. Mah, A. Nontji, M.K. Moosa. 1997. The Ecology of The Indonesian Seas. Part Two. Periplus Edition (HK) Ltd. Singapore

\section{To cite this article:}

Litaay, M., Deviana, M. \& Priosambodo, D. 2017. Biodiversity and Distribution of Gastropods at Seagrass Meadow of Balangdatu Waters Tanakeke Island South Sulawesi Indonesia. International Journal of Applied Biology. 1(2):58-66. 